\title{
Circulating Tumor Cells as Prognostic Marker in Japanese patients with Kras Wild-type Metastatic Colorectal Cancer Receiving Panitumumab after Progression on Cetuximab
}

Yoshihito Ohhara ${ }^{1,2}$, Satoshi Matsusaka ${ }^{1,3 *}$, Toshiyasu Watanabe1, Eiji Shinozaki ${ }^{1}$, Mitsukuni Suenaga ${ }^{1}$, Nobuyuki Mizunuma $^{1}$ and Kiyohik Hatake $^{3}$

${ }^{1}$ Department of Gastroenterology, Cancer Institute Hospital of Japanese Foundation for Cancer Research, Tokyo, Japan

${ }^{2}$ Department of Medical Oncology, Hokkaido University Graduate School of Medicine, Hokkaido, Japan

${ }^{3}$ Cancer Chemotherapy Center, Clinical Chemotherapy, Japanese Foundation for Cancer Research, Tokyo, Japan

\begin{abstract}
Background: Panitumumab has a high affinity for epidermal growth factor receptor. Its utility as a salvage therapy is unknown in cetuximab-resistant colorectal cancer. We assessed the prognostic and predictive role of circulating tumor cells (CTCs) in KRAS wild-type metastatic Colorectal Cancer (mCRC) patients treated with panitumumab after progression on cetuximab.

Methods: Panitumumab (6 mg/kg every 2 weeks) was administered as salvage therapy in a patient with cetuximab-resistant $\mathrm{mCRC}$. The CTC count in whole blood at baseline was determined using immunomagnetics.

Results: Nineteen patients were enrolled in the study between July 2010 and March 2011. Five patients had Stable Disease (SD) with a response rate of $0 \%$. Four patients reached long-SD, which was defined as continuous SD of more than 120 days. All of these four patients had $<2$ CTCs. Multivariate analysis revealed that $\geq 2$ CTCswas an independent predictor of Progression-Free Survival (PFS) (hazard ratio: 7.275, $P=0.013$ ). Patients with $\geq 2$ CTCs had a shorter median PFS than those with $<2$ CTCs (1.8 versus 3.6 months, $P=0.008$ ). No statistically significant difference was observed in median overall survival between patients with $\geq 2$ CTCs and those with <2 CTCs $(3.2$ versus 6.7 months, $P=0.164$ ).
\end{abstract}

Conclusions: These results suggest that $<2$ CTCs indicates that panitumumab may be effective in patients with KRAS-wild-type mCRC after progression on cetuximab.

Keywords: Cetuximab; Circulating tumor cells; Colorectal cancer; Panitumumab; Salvage therapy

\section{Introduction}

Colorectal cancer was the third most common malignancy and second most frequent cause of cancer-related death worldwide in 2008 [1]. The treatment options for patients with metastatic Colorectal Cancer (mCRC) have changed dramatically over the past few decades [2]. The standard chemotherapy regimen for patients with mCRC consists of fluoropyrimidine, irinotecan, and oxaliplatin, in combination or sequentially [3]. Furthermore, two types of monoclonal antibody drugs have recently been used in mCRC: bevacizumab, an antibody against vascular endothelial growth factor; and cetuximab or panitumumab, antibodies against Epidermal Growth Factor Receptor (EGFR), with the results showing an improvement in survival $[4,5]$.

Circulating Tumor Cells (CTCs) have been reported in peripheral blood in various cancers [6] and have been shown to be a predictor of Progression-Free Survival (PFS) and Overall Survival (OS) in patients with metastatic breast cancer $[7,8]$, prostate cancer [9], and gastric cancer [10]. Cohen et al. [11,12] reported that the number of CTCs at baseline and during treatment was an independent predictor of PFS and OS in patients with mCRC. Tol et al. [13] reported that the CTC count at baseline of treatment independently predicted PFS and OS in mCRC patients undergoing combination chemotherapy including cetuximab. Our previous study indicated that the CTC count after bevacizumab-containing chemotherapy predicted outcome in Japanese patients with mCRC [14]. Moreover, Kuboki et al. reported the utility of CTC count and EGFR expression on CTCs as biomarker for efficacy of cetuximab treatment for mCRC [15]. Taken together, these results clearly indicate that ethnicity exerts little effect on the reliability of CTC count as a predictor of PFS and OS in mCRC patients.
The role of EGFR inhibition in KRAS wild-type (WT; that is, non-mutated) mCRC has been established in systemic chemotherapy. Cetuximab is a chimeric antibody that consists of approximately $30 \%$ murine protein, while panitumumab is a fully human monoclonal antibody. Panitumumab has high affinity for EGFR [16]. This suggests that panitumumab might be effective in some patients with cetuximabrefractory KRAS-WT mCRC.

The purpose of this study was to investigate the prognostic role of CTCs for panitumumab treatment in KRAS-WT mCRC patients after progression on cetuximab.

\section{Materials and methods}

\section{Patients}

Circulating tumor cells were collected from patients with KRAS-WT mCRC being treated with panitumumab after progression on cetuximab at The Cancer Institute Hospital of the Japanese Foundation for Cancer

*Corresponding author: Satoshi Matsusaka, Department of Gastroenterology, Cancer Institute Hospital, Japanese Foundation for Cancer Research, 3-8-31 Ariake, Koto-ku, Tokyo, Japan, Tel: +81-3-3520-0111; Fax: +81-3-3570-0343; E-mail: satoshi.matsusaka@jfcr.or.jp

Received November 14, 2013; Accepted December 09, 2013; Published December 11, 2013

Citation: Ohhara Y, Matsusaka S, Watanabe T, Shinozaki E, Suenaga M, et al. (2013) Circulating Tumor Cells as Prognostic Marker in Japanese patients with Kras Wild-type Metastatic Colorectal Cancer Receiving Panitumumab afte Progression on Cetuximab. J Cytol Histol 5: 204. doi:10.4172/2157-7099.1000204

Copyright: $\odot 2013$ Ohhara Y, et al. This is an open-access article distributed unde the terms of the Creative Commons Attribution License, which permits unrestricted use, distribution, and reproduction in any medium, provided the original author and source are credited. 
Citation: Ohhara Y, Matsusaka S, Watanabe T, Shinozaki E, Suenaga M, et al. (2013) Circulating Tumor Cells as Prognostic Marker in Japanese patients with Kras Wild-type Metastatic Colorectal Cancer Receiving Panitumumab after Progression on Cetuximab. J Cytol Histol 5: 204. doi:10.4172/2157-7099.1000204

Page 2 of 5

Research (JFCR). Written informed consent for enrollment in the study was obtained from each patient. This protocol was approved by the institutional review board of the Cancer Institute Hospital of the JFCR. The eligibility criteria were as follows: (1) histologically proven mCRC; (2) received cetuximab-containing chemotherapy until disease-progression; (3) 18 years or older, with an Eastern Cooperative Oncology Group (ECOG) performance status (PS) of 0-2 at the start of panitumumab treatment; (4) adequate organ function; and (5) KRAS-WT.

\section{Treatment design and evaluation}

Patients received panitumumab $6 \mathrm{mg} / \mathrm{kg}$ intravenously every 2 weeks. Treatment continued until disease progression or unacceptable toxicity.

Tumor response was evaluated radiographically every 8 weeks using the response evaluation criteria in solid tumors (RECIST), version 1.0 [17]. Under panitumumab therapy, Progressive Disease (PD) was defined as clinical (e.g., increased ascites; obstructive jaundice or ileus due to peritoneal metastasis) or radiographic evidence of progression.

There were no limitations with regard to post-study treatment.

\section{Sample preparation for isolation of CTCs from blood and sample analysis}

For isolation of CTCs, $10-\mathrm{mL}$ samples of blood were drawn into a Cell Save Preservative Tube (Veridex LLC) prior to initiation of panitumumab treatment. The Cell Search system (Veridex LLC) consists of the Cell Prep system, the Cell Search Epithelial Cell Kit (for measurement of CTCs) and the Cell Spotter Analyzer. The Cell Prep system is a semi-automated sample preparation system, and the Cell Search Epithelial Cell kit consists of ferrofluids coated with epithelial cell-specific EpCAM antibodies to immunomagnetically enrich epithelial cells; a mixture of two phycoerythrin-conjugated antibodies that bind to cytokeratin 8,18 and 19; an antibody to CD45 conjugated to allophycocyanin; nuclear dye $4 \$$, 6-diamidino-2-phenylindole (DAPI) to fluorescently label the cell; and buffers to wash, permeabilize and resuspend the cells. Sample processing and evaluation were performed as described by Matsusaka et al. [14]. The criteria for an object to be defined as a CTC included round-to-oval morphology, a visible nucleus (DAPI-positive), positive staining for cytokeratin, and negative staining for CD45. Results of cell enumeration are always expressed as the number of cells per $7.5 \mathrm{~mL}$ blood.

\section{Data collection}

Factors included in the univariate analyses were as follows: age, sex, PS; previous primary site resection; advanced or recurrent, metastatic site (liver, lung, lymph node, and peritoneum); presence of measurable lesions; carcinoembryonic antigen, carbohydrate antigen 19-9; interval between cetuximab and panitumumab; CTCs.

\section{Statistical analysis}

Progression-free survival in panitumumab therapy was defined as the interval between the start of therapy to recognition of PD. Overall survival was defined as the interval between the start of panitumumab to death or the date of last follow-up. Survival data were estimated by the Kaplan-Meier method. Survival curves were compared using a log-rank test. Predictive factors were established by searching all variables that significantly influenced PFS or OS at a $P$-value of $<0.2$ in the univariate analysis. Multivariate analysis was carried out using the Cox proportional hazards regression model. A $P$-value of $<0.05$ was considered statistically significant.

\section{Results}

\section{Patient characteristics}

Nineteen patients were enrolled in the study between July 2010 and March 2011 at the Cancer Institute Hospital, Japan. Patient characteristics are shown in Tables $1 \mathrm{a}$ and $1 \mathrm{~b}$.

The median age was 54 years, with a range of $40-78$ years. Fortyseven percent of the patients were male. Only one patient had an ECOG PS of 2. Fifteen patients (78.9\%) had two or more sites of metastasis.

Eighteen patients $(94.7 \%)$ received regimens containing oxaliplatin and irinotecan, and 13 patients $(68.4 \%)$ received regimens containing bevacizumab as a prior chemotherapy. The median number of prior regimens was three.

Among the total number of 19 patients, the objective Response Rate (RR) was $0 \%$. Five patients had Stable Disease (SD), of whom 4 achieved long-SD, defined as continuous SD of more than 120 days. All four patients had $<2$ CTCs.

\section{Univariate and multivariate analyses of predictors of PFS}

Univariate and multivariate Cox proportional-hazards regression was used to assess the association between factors of interest and PFS or OS. In the univriate analysis, the presence of primary site, lymph node metastasis, and CTC level at the start of panitumumab predicted PFS. The results showed that $\geq 2$ CTCs was a stronger predictor than other numbers of CTCs, as ascertained by comparing each CTC level (Table 2). To evaluate the independent predictive effect of panitumumab, a multivariate Cox regression analysis was carried out (Table 3 ). The results

\begin{tabular}{|c|c|c|c|}
\hline \multirow{2}{*}{\multicolumn{2}{|c|}{ Characteristics }} & \multirow[t]{2}{*}{$n=19$} & \multirow[t]{2}{*}{$\%$} \\
\hline & & & \\
\hline \multicolumn{2}{|c|}{ Median age, years (range) } & 54 & $(40-78)$ \\
\hline \multicolumn{2}{|l|}{ Sex, male/female } & $9 / 10$ & \\
\hline \multicolumn{2}{|c|}{ ECOG performance status: 0/1/2 } & $9 / 9 / 1$ & \\
\hline \multicolumn{2}{|c|}{ Primary site: Colon/Rectum } & $8 / 11$ & \\
\hline \multicolumn{2}{|c|}{ Advanced/Recurrence } & $9 / 10$ & \\
\hline \multirow[t]{5}{*}{ Metastasis site: } & Liver & 11 & $(57.9 \%)$ \\
\hline & Lung & 11 & $(57.9 \%)$ \\
\hline & Lymph Nodes & 10 & $(52.6 \%)$ \\
\hline & Peritoneum & 7 & $(36.8 \%)$ \\
\hline & Other & 7 & $(36.8 \%)$ \\
\hline \multicolumn{2}{|c|}{ Metastasis sites $>2$} & 15 & $(78.9 \%)$ \\
\hline \multicolumn{2}{|c|}{ Median number of prior chemotherapies (range) } & 3 & $(1-5)$ \\
\hline \multicolumn{2}{|c|}{ Oxaliplatin-containing regimen } & 18 & $(94.7 \%)$ \\
\hline \multicolumn{2}{|c|}{ Irinotecan-containing regimen } & 18 & $(94.7 \%)$ \\
\hline \multicolumn{2}{|c|}{ Bevacizumab-containing regimen } & 13 & $(68.4 \%)$ \\
\hline \multicolumn{2}{|c|}{ Cetuximab-containing regimen } & 19 & $(100 \%)$ \\
\hline \multicolumn{4}{|l|}{ Outcome } \\
\hline \multicolumn{2}{|c|}{ Complete response } & 0 & $(0 \%)$ \\
\hline \multicolumn{2}{|c|}{ Partial response } & 0 & $(0 \%)$ \\
\hline \multicolumn{2}{|c|}{ Stable disease (SD) } & 5 & $(29.2 \%)$ \\
\hline \multicolumn{2}{|c|}{ Progressive disease } & 12 & $(54.2 \%)$ \\
\hline \multicolumn{2}{|l|}{ Not evaluable } & 2 & $(12.5 \%)$ \\
\hline \multicolumn{2}{|l|}{ Long SD } & 4 & $(21.1 \%)$ \\
\hline
\end{tabular}

Long SD: stable disease for more than 120 days.

Table 1(a): Patient characteristics and treatment response. 
Citation: Ohhara Y, Matsusaka S, Watanabe T, Shinozaki E, Suenaga M, et al. (2013) Circulating Tumor Cells as Prognostic Marker in Japanese patients with Kras Wild-type Metastatic Colorectal Cancer Receiving Panitumumab after Progression on Cetuximab. J Cytol Histol 5: 204. doi:10.4172/2157-7099.1000204

Page 3 of 5

\begin{tabular}{|c|c|c|c|c|c|c|c|}
\hline Case & Age & Sex & $\begin{array}{c}\text { PFS } \\
\text { (months) }\end{array}$ & $\begin{array}{c}\text { Interval } \\
\text { OS }\end{array}$ & $\begin{array}{c}\text { Inths) } \\
\text { between Cmab } \\
\text { and Pmab } \\
<120 \text { days }\end{array}$ & $\begin{array}{c}\text { Best } \\
\text { overall } \\
\text { response }\end{array}$ & $\begin{array}{c}\text { CTC } \\
\text { count }\end{array}$ \\
\hline 1 & 64 & $\mathrm{~F}$ & 0.4 & 1.7 & Yes & PD & 19 \\
\hline 2 & 45 & $\mathrm{~F}$ & 2.3 & 4.3 & Yes & PD & 21 \\
\hline 3 & 54 & $\mathrm{~F}$ & 3.0 & 6.4 & Yes & PD & 1 \\
\hline 4 & 43 & $\mathrm{M}$ & 4.5 & 17.6 & Yes & SD & 0 \\
\hline 5 & 48 & $\mathrm{M}$ & 1.0 & 1.9 & Yes & PD & 18 \\
\hline 6 & 40 & $\mathrm{~F}$ & 3.0 & 4.9 & Yes & PD & 0 \\
\hline 7 & 40 & $\mathrm{M}$ & 1.8 & 3.2 & Yes & PD & 2 \\
\hline 8 & 57 & $\mathrm{M}$ & 0.5 & 2.2 & No & NE & 12 \\
\hline 9 & 74 & $\mathrm{~F}$ & 5.3 & 10.7 & No & SD & 0 \\
\hline 10 & 60 & $\mathrm{~F}$ & 3.6 & 7.1 & No & SD & 0 \\
\hline 11 & 58 & $\mathrm{M}$ & 3.5 & 11.2 & Yes & SD & 9 \\
\hline 12 & 47 & $\mathrm{~F}$ & 2.1 & 5.2 & Yes & PD & 2 \\
\hline 13 & 41 & $\mathrm{M}$ & 2.0 & 3.5 & Yes & PD & 0 \\
\hline 14 & 59 & $\mathrm{M}$ & 4.6 & 12.8 & No & SD & 0 \\
\hline 15 & 71 & $\mathrm{~F}$ & 1.4 & 4.8 & Yes & PD & 0 \\
\hline 16 & 78 & $\mathrm{~F}$ & 0.7 & 10.3 & Yes & NE & 4 \\
\hline 17 & 54 & $\mathrm{M}$ & 1.4 & 3.1 & Yes & PD & 222 \\
\hline 18 & 71 & $\mathrm{~F}$ & 4.1 & 6.7 & Yes & PD & 1 \\
\hline 19 & 61 & $\mathrm{M}$ & 2.6 & 9.0 & Yes & PD & 0 \\
\hline
\end{tabular}

PFS=Progression-Free Survival; OS=Overall Survival; $C T C=$ Circulating Tumor Cells; $\mathrm{SD}=$ Stable Disease; $\mathrm{PD}=$ Progressive Disease; NE=Not Evaluable; $\mathrm{Cmab}=$ Cetuximab; Pmab=Panitumumab.

*Interval between last administration of cetuximab and first administration of panitumumab.

Blood sample for CTC count was taken on same day as administration of panitumumab.

Table 1(b): Relationship between clinical outcome and CTC count.

\begin{tabular}{|c|c|c|c|c|c|c|}
\hline \multirow{2}{*}{ Parameter } & \multicolumn{3}{|c|}{ PFS } & \multicolumn{3}{|c|}{ os } \\
\hline & HR & $95 \% \mathrm{Cl}$ & $P$-value & HR & $95 \% \mathrm{Cl}$ & $P$-value \\
\hline Age $>60$ & 0.57 & $0.176-1.849$ & 0.35 & 1.133 & $0.372-3.455$ & 0.826 \\
\hline $\begin{array}{l}\text { Sex: male vs. } \\
\text { female }\end{array}$ & 0.8 & $0.469-1.365$ & 0.413 & 1.22 & $0.688-2.164$ & 0.496 \\
\hline$P S \geq 1$ & 1.352 & $0.439-4.162$ & 0.599 & 3.872 & $1.164-12.877$ & 0.027 \\
\hline $\begin{array}{l}\text { Presence of } \\
\text { primary site }\end{array}$ & 3.756 & $0.721-19.559$ & 0.116 & 1.817 & $0.21-15.706$ & 0.587 \\
\hline $\begin{array}{l}\text { Advanced } \\
\text { vsrecurrence }\end{array}$ & 0.803 & $0.47-1.371$ & 0.421 & 1.008 & $0.605-1.678$ & 0.977 \\
\hline Liver metastasis & 0.477 & $0.133-1.71$ & 0.256 & 0.946 & $0.325-2.756$ & 0.919 \\
\hline Lung metastasis & 1.641 & $0.504-5.338$ & 0.411 & 0.395 & $0.128-1.216$ & 0.105 \\
\hline $\begin{array}{l}\text { Lymph node } \\
\text { metastasis }\end{array}$ & 2.231 & $0.72-6.914$ & 0.164 & 0.944 & $0.337-2.642$ & 0.913 \\
\hline $\begin{array}{l}\text { Peritoneal } \\
\text { metastasis }\end{array}$ & 0.927 & $0.282-3.046$ & 0.9 & 2.243 & $0.721-6.978$ & 0.163 \\
\hline CEA $>10 \mathrm{ng} / \mathrm{ml}$ & 1.02 & $0.22-4.726$ & 0.979 & 3.031 & $0.377-24.346$ & 0.297 \\
\hline CA19-9>100 U/ml & 1.287 & $0.429-3.864$ & 0.653 & 1.778 & $0.556-5.69$ & 0.332 \\
\hline $\begin{array}{l}\text { Interval between } \\
\text { Cmab and } \\
\text { Pmab<120 days }\end{array}$ & 0.011 & $0-2.052$ & 0.091 & 0.407 & $0.129-1.287$ & 0.126 \\
\hline $\mathrm{CTC} \geq 1$ & 3.277 & $0.964-11.144$ & 0.057 & 2.467 & $0.81-7.51$ & 0.112 \\
\hline $\mathrm{CTC} \geq 2$ & 5.017 & $1.366-18.427$ & 0.015 & 2.097 & $0.724-6.075$ & 0.173 \\
\hline $\mathrm{CTC} \geq 3$ & 3.268 & $0.913-11.698$ & 0.069 & 2.091 & $0.714-6.122$ & 0.178 \\
\hline
\end{tabular}

$\mathrm{HR}=$ Hazard Ratio; $\mathrm{Cl}=$ Confidence Interval; $\mathrm{PS}=$ Performance Status;

CEA=CarcinoEmbryonic Antigen; CA19-9=Carbohydrate Antigen 19-9;

Cmab=cetuximab; Pmab=Panitumumab; CTC=Circulating Tumor Cells:

PFS=Progression-Free Survival; OS=Overall Survival.

Table 2: Univariate analysis for PFS and OS.

revealed that $\geq 2$ CTCs showed an independent predictive role (hazard ratio; 7.275, 95\% Confidence Interval [CI], 1.515-34.931, $P=0.013$ ).

\section{Univariate and multivariate analyses of predictors of OS}

Univariate analysis revealed 4 factors which were associated with poor survival: PS $\geq 1$, lung metastasis, peritoneal metastasis, and CTC level at the start of panitumumab (Table 2). We adopted $\geq 2$ CTCs in order to match the CTC levels in PFS. Multivariate analysis, however, showed no statistical significance among them (Table 3 ).

\section{Analysis of PFS and OS according to CTC level}

At the time of analysis, 16 patients (84.2\%) had died from disease progression. Figure 1a shows the Kaplan-Meier plots for prediction of PFS using the CTC count at start of panitumumab treatment. Patients with $\geq 2$ CTCs had a shorter median PFS (1.8 months; 95\% CI, 1.0-2.7) than those with $<2$ CTCs (3.6 months; 95\% CI, 2.0-5.2) ( $P=0.008)$.

Figure 1b shows the Kaplan-Meier plots for prediction of OS using the CTC count at start of panitumumab treatment. There was no significant difference in median OS between patients with $\geq 2$ CTCs $(3.2$ months; 95\% CI, 2.9-3.5) and those with <2 CTCs (6.7 months; 95\% CI, 5.6-7.9) $(P=0.164)$. However, patients with $\geq 2$ CTCs showed a trend toward a shorter OS.

\section{Discussion}

The value of panitumumab as a salvage agent in cetuximabresistant mCRC is unknown. The purpose of this study was to determine the value of CTCs as a surrogate marker in salvage therapy with panitumumab in patients with cetuximab-refractory KRASWT mCRC. In the PANERB trial, Metges et al. reported an objective response rate of $22 \%$ to panitumumab after progression on cetuximab plus irinotecan [18]. Moreover, they showed that the disease control rate (objective response and SD) was $73 \%$ in patients who had responded to cetuximab and irinotecan previously. In contrast, in January 2011,

\begin{tabular}{|l|l|c|c|c|}
\hline Parameter & & HR & $95 \% \mathrm{Cl}$ & $P$-value \\
\hline PFS & & & & \\
\hline & Presence of primary site & 0.707 & $0.101-4.936$ & 0.727 \\
\hline & Lymph node metastasis & 3.125 & $0.859-11.374$ & 0.084 \\
\hline & CTC $\geq 2$ & 7.275 & $1.515-34.931$ & 0.013 \\
\hline OS & & & & \\
\hline & PS $\geq 1$ & 2.256 & $0.467-10.888$ & 0.311 \\
\hline & Lung metastasis & 0.57 & $0.163-1.989$ & 0.378 \\
\hline & Peritoneal metastasis & 1.378 & $0.315-6.019$ & 0.67 \\
\hline & CTC $\geq 2$ & 2.017 & $0.59-6.898$ & 0.263 \\
\hline
\end{tabular}

Table 3: Multivariate analysis for PFS and OS.
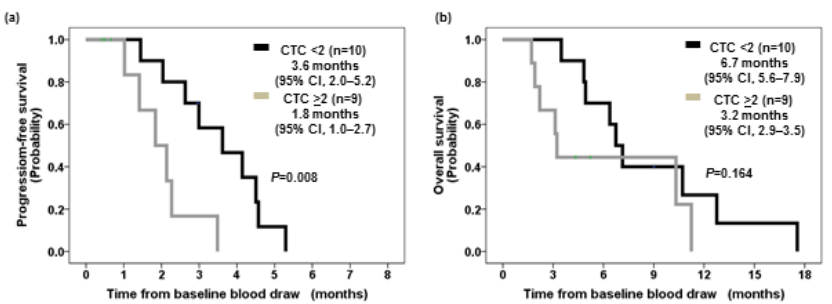

Figure 1: Kaplan-Meyer estimates of progression-free survival (a) and overall survival (b), according to patients with less than two circulating tumor cells (CTCs, black line) or $\geq 2$ CTCs (gray line) at the start of panitumumab treatment. 
Citation: Ohhara Y, Matsusaka S, Watanabe T, Shinozaki E, Suenaga M, et al. (2013) Circulating Tumor Cells as Prognostic Marker in Japanese patients with Kras Wild-type Metastatic Colorectal Cancer Receiving Panitumumab after Progression on Cetuximab. J Cytol Histol 5: 204. doi:10.4172/2157-7099.1000204

Wadlow et al. reported that panitumumab was not active as a salvage therapy in patients with cetuximab-resistant KRAS-WT mCRC $[19,20]$. At the time of that report, the RR in the present study was $0 \%$. Therefore, we had to stop our study and assess data obtained from a total of 19 patients. It is impossible to determine whether all patients who are cetuximab-refractory would benefit from salvage therapy with panitumumab based on the results of the Wadlow study alone. Our goal here was to determine whether CTCs might act as such an indicator.

The results of the present study clearly show that consistent achievement of $<2$ CTCs at start of panitumumab after progression on cetuximab is a strong indicator that patients treated with panitumumab will have better PFS, whereas $\geq 2$ CTCs is a strong indicator that those will probably confer no benefit of panitumumab treatment. These data suggest that the CTC count is valuable in identifying patients who will benefit from panitumumab after failure of cetuximab. We were unable, however, to determine the difference in potential survival benefit between patients with $<2$ CTCs and those with $\geq 2$ CTCs, probably because we had to stop the study earlier than expected, leaving a total of only 19 patients on whom to base an assessment. The limitation of our study was too small sample size and this study was under power to detect the difference in OS between two groups. To clarify the utility of CTCs in panitumumab treatment, we will need to undergo the prospective study in patients with cetuximab-refractory KRAS-WT mCRC between patients with $<2$ CTCs treated with panitumumab plus BSC and those with BSC only.

The previously reported lower concordance levels of KRAS between the primary tumor and metastases probably resulted from falsenegative results in underpowered studies and the incorrect evaluation of the amount of tumor tissue in the sample and the sensitivity of testing methods. Previously published data showed that a considerable fraction of colorectal lymph node metastases did not necessarily correspond with the primary tumor in terms of KRAS mutation status [21]. Heterogeneity in lymph node metastases could explain this discordance in a few patients, but its main mechanism is unknown. Misale $S$ et al. reported that use of anti-EGFR drugs for mCRC contributed to acquisition of a KRAS mutation. They offered two possible explanations for the discordant results of KRAS: heterogeneity of KRAS status within the primary tumor; and clonal selection during the process of metastasis. In clinical practice, they identified 10 patients with acquired resistance to anti-EGFR therapy who were KRAS-WT by this technique. These techniques identified the KRASG13D mutation in four samples, and the simultaneous presence of G12D and G13D mutations in one case. In the six patients for whom sufficient pre-treatment tumor samples were available for high coverage 454 sequence analyses or BEAMing, KRAS mutations were found to be absent at pre-treatment. Tumors from a further eight patients treated with cytotoxic chemotherapy but not previously exposed to anti-EGFR therapies were also analyzed by 454 deep sequencing. No evidence of KRAS mutation was identified in all eight cases. These results indicate that treatment with antiEGFR antibodies but not cytotoxic chemotherapy is associated with the acquisition of KRAS mutations [22]. Recently, the mechanism of resistance to anti-EGFR therapy is being clarified [23]. Montagut C et al. reported an acquired EGFR ectodomain mutation (S492R) that prevented cetuximab binding, thereby conferring resistance to the drug [24]. However, the S492R mutation cells retained binding to panitumumab, resulting in inhibition of growth. In this study, two of ten patients acquired this mutation after cetuximab treatment. One patient with cetuximab resistance harboring the S492R mutation responded to treatment with panitumumab. This suggests that panitumumab may be effective in patients with the S492R mutation after failure of cetuximab. A re-biopsy needs to be performed before commencing treatment with molecularly targeted drugs. In future research, one of our goals is to analyze KRAS status using CTCs, which could potentially play a core role in liquid biopsy.

In conclusion, the present results suggest that CTC count prior to initiation of panitumumab may be useful todetermine the better prognosis patients with KRAS-WT mCRC after progression on cetuximab.

\section{Acknowledgements}

We wish to thank Sayuri Minowa, Harumi Shibata, and Mariko Mikuniya for their excellent technical assistance.

\section{References}

1. http://globocan.jarc.fr/factsheets/cancers/colorectal.asp.

2. Punt CJ (2004) New options and old dilemmas in the treatment of patients with advanced colorectal cancer. Ann Oncol 15: 1453-1459.

3. Koopman M, Antonini NF, Douma J, Wals J, Honkoop AH, et al. (2007) Sequential versus combination chemotherapy with capecitabine, irinotecan and oxaliplatin in advanced colorectal cancer (CAIRO): a phase III randomised controlled trial. Lancet 370: 135-142.

4. Saltz LB, Clarke S, Díaz-Rubio E, Scheithauer W, Figer A, et al. (2008) Bevacizumab in combination with oxaliplatin-based chemotherapy as first-line therapy in metastatic colorectal cancer: a randomized phase III study. J Clin Oncol 26: 2013-2019.

5. Bokemeyer C, Bondarenko I, Makhson A, Hartmann JT, Aparicio J, et al. (2009) Fluorouracil, leucovorin, and oxaliplatin with and without cetuximab in the firstline treatment of metastatic colorectal cancer. J Clin Oncol 27: 663-671.

6. Allard WJ, Matera J, Miller MC, Repollet M, Connelly MC, et al. (2004) Tumor cells circulate in the peripheral blood of all major carcinomas but not in healthy subjects or patients with nonmalignant diseases. Clin Cancer Res 10: 68976904.

7. Cristofanilli M, Budd GT, Ellis MJ, Stopeck A, Matera J, et al. (2004) Circulating tumor cells, disease progression, and survival in metastatic breast cancer. $\mathrm{N}$ Engl J Med 351: 781-791.

8. Dawood S, Broglio K, Valero V, Reuben J, Handy B, et al. (2008) Circulating tumor cells in metastatic breast cancer: from prognostic stratification to modification of the staging system? Cancer 113: 2422-2430.

9. Danila DC, Heller G, Gignac GA, Gonzalez-Espinoza R, Anand A, et al. (2007) Circulating tumor cell number and prognosis in progressive castration-resistant prostate cancer. Clin Cancer Res 13: 7053-7058

10. Matsusaka S, Chìn K, Ogura M, Suenaga M, Shinozaki E, et al. (2010) Circulating tumor cells as a surrogate marker for determining response to chemotherapy in patients with advanced gastric cancer. Cancer Sci 101: 10671071.

11. Cohen SJ, Punt CJ, lannotti N, Saidman BH, Sabbath KD, et al. (2009) Prognostic significance of circulating tumor cells in patients with metastatic colorectal cancer. Ann Oncol 20: 1223-1229.

12. Cohen SJ, Punt CJ, lannotti N, Saidman BH, Sabbath KD, et al. (2008) Relationship of circulating tumor cells to tumor response, progression-free survival, and overall survival in patients with metastatic colorectal cancer. $\mathrm{J}$ Clin Oncol 26: 3213-3221.

13. Tol J, Koopman M, Miller MC, Tibbe A, Cats A, et al. (2010) Circulating tumour cells early predict progression-free and overall survival in advanced colorectal cancer patients treated with chemotherapy and targeted agents. Ann Oncol 21: 1006-1012.

14. Matsusaka S, Suenaga M, Mishima Y, Kuniyoshi R, Takagi K, et al. (2011) Circulating tumor cells as a surrogate marker for determining response to chemotherapy in Japanese patients with metastatic colorectal cancer. Cancer Sci 102: 1188-1192.

15. Kuboki Y, Matsusaka S, Minowa S, Shibata H, Suenaga M, et al. (2013) Circulating tumor cell (CTC) count and epithelial growth factor receptor 
Citation: Ohhara Y, Matsusaka S, Watanabe T, Shinozaki E, Suenaga M, et al. (2013) Circulating Tumor Cells as Prognostic Marker in Japanese patients with Kras Wild-type Metastatic Colorectal Cancer Receiving Panitumumab after Progression on Cetuximab. J Cytol Histol 5: 204. doi:10.4172/2157-7099.1000204

Page 5 of 5

expression on CTCs as biomarkers for cetuximab efficacy in advanced colorectal cancer. Anticancer Res 33: 3905-3910.

16. Cohenuram M, Saif MW (2007) Panitumumab the first fully human monoclonal antibody: from the bench to the clinic. Anticancer Drugs 18: 7-15.

17. Therasse P, Arbuck SG, Eisenhauer EA, Wanders J, Kaplan RS, et al. (2000) New guidelines to evaluate the response to treatment in solid tumors. European Organization for Research and Treatment of Cancer, National Cancer Institute of the United States, National Cancer Institute of Canada. J Natl Cancer Inst 92: $205-216$

18. Metges J, Raoul J, Achour N (2011) PANERB study: Panitumumab after cetuximab-based regimen failure. J Clin Oncol 28: 14000.

19. Wadlow RC, Hezel AF, Wolpin BM (2011) A single-arm trial of panitumumab in cetuximab refractory KRAS wild-type colorectal cancer. J Clin Oncol 29: 428.

20. Wadlow RC, Hezel AF, Abrams TA, Blaszkowsky LS, Fuchs CS, et al.
(2012) Panitumumab in patients with KRAS wild-type colorectal cancer after progression on cetuximab. Oncologist 17: 14.

21. Baldus SE, Schaefer KL, Engers R, Hartleb D, Stoecklein NH, et al. (2010) Prevalence and heterogeneity of KRAS, BRAF, and PIK3CA mutations in primary colorectal adenocarcinomas and their corresponding metastases. Clin Cancer Res 16: 790-799.

22. Misale S, Yaeger R, Hobor S, Scala E, Janakiraman M, et al. (2012) Emergence of KRAS mutations and acquired resistance to anti-EGFR therapy in colorectal cancer. Nature 486: 532-536.

23. Diaz LA Jr, Williams RT, Wu J, Kinde I, Hecht JR, et al. (2012) The molecular evolution of acquired resistance to targeted EGFR blockade in colorectal cancers. Nature 486: 537-540.

24. Montagut C, Dalmases A, Bellosillo B, Crespo M, Pairet S, et al. (2012) Identification of a mutation in the extracellular domain of the Epidermal Growth Factor Receptor conferring cetuximab resistance in colorectal cancer. Nat Med 18: 221-223. 